\title{
Lipid accumulation product as a marker of cardiometabolic susceptibility in women with different phenotypes of polycystic ovary syndrome
}

\author{
Ivana Božić-Antić1, Dušan Ilićc , Jelica Bjekić-Macut², Tamara Bogavac'1, Danijela Vojnović-Milutinović ${ }^{3}$ \\ Biljana Kastratovic-Kotlica ${ }^{4}$, Nataša Milić ${ }^{5}$, Olivera Stanojlovićc ${ }^{\text {, Zoran Andrić }}{ }^{\mathbf{2}}$ and Djuro Macut ${ }^{1}$
}

${ }^{1}$ Clinic of Endocrinology, Diabetes and Metabolic Diseases, Clinical Centre of Serbia, ${ }^{2}$ UMC Bežanijska kosa, Faculty of Medicine, ${ }^{3}$ Institute for Biological Research "Siniša Stanković", ${ }^{4} \mathrm{Clinic}$ of Obstetrics and Gynecology, Clinical Centre of Serbia, 5 Institute of Medical Statistics, and ${ }^{6}$ Institute of Medical Physiology, Faculty of Medicine, University of Belgrade, Belgrade, Serbia
Correspondence should be addressed to D Macut Email

djmacut@gmail.com

\begin{abstract}
Objective: There are limited data on cardiometabolic risk factors and the prevalence of metabolic syndrome (MetS) across the different PCOS phenotypes in Caucasian population. Lipid accumulation product (LAP) is a clinical surrogate marker that could be used for evaluation of MetS in clinical practice. The aim of the study was to analyze metabolic characteristics and the ability of LAP to predict MetS in different PCOS phenotypes.

Design: Cross-sectional clinical study analyzing 365 women with PCOS divided into four phenotypes according to the ESHRE/ASRM criteria, and 125 healthy BMI-matched controls.

Methods: In all subjects, LAP was determined and MetS was diagnosed according to the National Cholesterol Education Program/Adult Treatment Panel III (NCEP-ATP III), the International Diabetes Federation (IDF) and the Joint Interim Statement (JIS) criteria. Logistic regression and ROC curve analyses were used to determine predictors of MetS in each PCOS phenotype. All analyses were performed with age and BMI adjustment.

Results: All PCOS phenotypes in comparison to controls had higher prevalence of MetS assessed by NCEP-ATP III criteria, and only classic phenotypes when IDF and JIS criteria were used. All phenotypes had the same prevalence of MetS irrespective of used definition. LAP and exhibited the highest diagnostic accuracy and was an independent predictor of MetS in all phenotypes.

Conclusion: LAP is an independent and accurate clinical determinant of MetS in all PCOS phenotypes in our Caucasian population. All PCOS phenotypes, including non-classic ones, are metabolically challenged and with cardiovascular risk, particularly phenotype B.
\end{abstract}

\section{Introduction}

Polycystic ovary syndrome (PCOS) is a multisystem endocrinopathy that affects $15-25 \%$ reproductiveaged women according to ESHRE/ASRM criteria, and is characterized by various reproductive, obstetric and cardiometabolic features. ESHRE/ASRM definition considers polycystic ovary morphology (PCOM), oligo/anovulation (ANOV) and hyperandrogenism (HA) as equal criteria (1). When combined, these criteria form four different phenotypes: A (ANOV, HA, PCOM), B (ANOV, HA), C (HA, PCOM) and D (ANOV, 
PCOM). Several studies that have so far analyzed PCOS phenotypes have found differences in anthropometric, hormonal and metabolic characteristics between PCOS phenotypes (2-5). This implies different cardiometabolic risk factors in different PCOS phenotypes as well. A common conclusion is that phenotypes $\mathrm{A}$ and $\mathrm{B}$ have a higher metabolic risk than other phenotypes (6-8), but the data are inconsistent.

Frequent metabolic complications of PCOS are obesity, insulin resistance, dyslipidemia, dysglycemia, hypertension and the consequent development of low-grade inflammation (9). These cardiovascular risk factors are considered as independent predictors of cardiovascular morbidity and mortality (9) and usually cluster together into metabolic syndrome (MetS). Because of the high cost of treatment of associated morbidities (10), PCOS not only has significant health implications per se, but it also represents a significant economic burden on the general healthcare. However, patients with PCOS are still rarely evaluated for presence of metabolic and cardiovascular risk factors in routine clinical practice (11), and a long-term follow-up of cardiovascular outcomes is missing. One possible explanation could be that the procedures for its evaluation are both timeand money-consuming. Specifically, the evaluation of the presence of MetS, regardless of the MetS definition used should include measuring waist circumference (WC), blood pressure, high-density cholesterol (HDL), triglycerides, fasting glucose, as well as a 2-h oral glucose tolerance test (OGTT) (12). The additional problem is related to the lack of recommendation on which MetS definition should be used with specific populations, like with PCOS. Therefore, there is a need for a simple and accurate method for the assessment of cardiometabolic risk in PCOS women.

Lipid accumulation product (LAP) represents a clinical index based on the assessment of waist circumference (WC) and serum triglycerides (TG), which estimates visceral lipid accumulation. LAP is considered to be an independent predictor of metabolic syndrome (MetS), cardiovascular diseases and type 2 diabetes mellitus (T2DM) in healthy population $(13,14)$. In women with PCOS, LAP was shown to be a marker of increased cardiovascular risk (15), and was associated with impaired glucose tolerance (IGT) (16), MetS (17), and non-alcoholic fatty liver disease (NAFLD) (18). Recently, we have shown a strong association between LAP and MetS in Caucasian PCOS population. Moreover, LAP had the highest diagnostic accuracy in comparison to other
MetS determinants regardless of which MetS definition was used (NCEP-ATP III, IDF or JIS) (19). Since there is emerging evidence of variability of cardiometabolic risks in different PCOS phenotypes $(8,20)$, the aim of this study was to evaluate the metabolic characteristics and prevalence of MetS, as well as to analyze the ability of LAP to predict MetS in different phenotypes of women with PCOS.

\section{Subjects and methods}

\section{Subjects}

We analyzed 365 women with PCOS (age: $25.48 \pm 5.21$ years, BMI: $25.05 \pm 6.24 \mathrm{~kg} / \mathrm{m}^{2}$ ) and 125 healthy, BMI matched women (age: $30.35 \pm 5.62$ years, BMI: $25.41 \pm 5.16 \mathrm{~kg} / \mathrm{m}^{2}$ ). Subjects were referred to the outpatient endocrine clinic for evaluation of irregular menstrual cycles, infertility, obesity, hirsutism or acne. PCOS was defined according to the ESHRE/ASRM definition that requires the presence at least two of the following three criteria: (1) oligo/ amenorrhea or anovulation; (2) clinical and/or biochemical hyperandrogenism; and (3) morphologically polycystic ovaries on ultrasound (21). Clinical hyperandrogenism (hirsutism) was evaluated by Ferriman-Galway (FG) score system (22), and was considered to be present when FG score was $\geq 8$, irrespective of the concomitant presence of acne or alopecia (23). Free androgen index (FAI) was calculated by the formula ((testosterone $\times 100) / \mathrm{SHBG}$ ) with both testosterone and SHBG expressed in nmol/L (24). Biochemical hyperandrogenism (hyperandrogenemia) was defined by presence of FAI $>6$ and/or serum total testosterone (TT) $>2 \mathrm{nmol} / \mathrm{L}$. These cut-off values were based on examination of 56 non-selected, non-hirsute women presenting for routine controls who had regular menstrual cycles, and morphologically normal ovaries (25). Concomitantly, these cut-offs also corresponded to 95\% percentile of FAI and serum TT of analyzed healthy control women in this study. In all subjects, we excluded the presence of non-classical 21-hydroxylase deficiency, hyperprolactinemia, Cushing's disease, hypothyroidism, diabetes mellitus and history of drug or alcohol abuse. No patients had received any hormone treatment for at least 3 months before the study.

Control group of healthy women were volunteers referred after regular yearly gynecological examination. They were normoandrogenic, without hirsutism, had regular, ovulating menstrual cycles and morphologically normal ovaries on ultrasound. 


\section{Methods}

In all subjects, we measured systolic and diastolic blood pressure (SBP and DBP, respectively), and waist circumference (WC) at the level of umbilicus. Body mass index (BMI, $\mathrm{kg} / \mathrm{m}^{2}$ ) was calculated by formula: weight $(\mathrm{kg}) /$ height $(\mathrm{m})^{2}$. After a 12 -h overnight fast, in the follicular phase of the menstrual cycle (between days 3 and 7) for women with regular menstrual cycles and irrespective on the menstrual cycle for those with oligo/ amenorrhea, blood samples were taken for determination of total cholesterol (TC), high-density lipoprotein (HDL), low-density lipoprotein (LDL), triglycerides (TG), fasting plasma glucose (FPG), insulin, serum total testosterone (TT), sex-hormone-binding protein (SHBG). Samples for hormonal analyses were frozen at $-80^{\circ} \mathrm{C}$ until measurement. In all subjects, 2-h oral glucose tolerance test (OGTT) with $75 \mathrm{~g}$ glucose was performed on the second day of examination, and serum glucose was determined at $0 \mathrm{~min}$ and $120 \mathrm{~min}$ of the test. Serum glucose $(\mathrm{mmol} / \mathrm{L})$ was measured by the glucose-oxidase method (Randox, UK). TC $(\mathrm{mmol} / \mathrm{L})$ and TG $(\mathrm{mmol} / \mathrm{L})$ were determined using standard enzymatic methods (TC: cholesterol oxidase, Randox, TG: glycerol-3-phosphat oxidase, Randox). HDL (mmol/L) was measured by direct method (Randox), and LDL $(\mathrm{mmol} / \mathrm{L})$ determined by the Friedewald formula (26). Serum insulin (mU/L) concentrations were determined by radioimmunoassay (RIA INSULIN (PEG), INEP, Belgrade, Serbia, intra- and interassay CV were 2.5 and $7.7 \%$, respectively). Serum TT (nmol/L) was measured by radioimmunoassay (TESTOCT2, CIS Bio international, Gifsur-Yvette Cedex, France; intra- and interassay CVs 4.5 and $5.1 \%$, respectively). SHBG (nmol/L) was measured by radioimmunoassay (SHBG-RIACT, CIS Bio international, Gifsur-Yvette Cedex; intra- and interassay CVs were 3.9 and $4.7 \%$, respectively). Insulin resistance (IR) was estimated by the homeostasis model assessment of IR (HOMAIR= insulin $(\mathrm{mIU} / \mathrm{L}) \times$ glucose $(\mathrm{mmol} / \mathrm{L}) / 22.5)(27)$. LAP was calculated using formula: ((WC-58) $\times$ triglycerides) (28), where 58 is the minimum WC value for women according to the Third National Health and Nutrition Examination Survey (NHANES III). All subjects were assessed as having MetS using three different international criteria: NCEPATP III (29), IDF (30) and Joint Interim Statement (JIS) criteria (31). According to NCEP-ATP III, the diagnosis of MetS was established if any three or more of the following criteria were satisfied: 1) WC: $\geq 88 \mathrm{~cm}, 2$ ) triglycerides (TG) $\geq 1.7 \mathrm{mmol} / \mathrm{L}, 3) \mathrm{SBP} \geq 130$ and/or DBP $\geq 85 \mathrm{mmHg}$, 4) fasting $\mathrm{HDL}<1.3 \mathrm{mmol} / \mathrm{L}$, and 5) fasting plasma glucose $(\mathrm{FPG}) \geq 6.1 \mathrm{mmol} / \mathrm{L}$. The IDF definition of the MetS for the Europid population considered central adiposity (defined as WC $80 \mathrm{~cm}$ ) as an obligatory factor for the diagnosis of the MetS, plus two of the following criteria: $\mathrm{TG} \geq 1.7 \mathrm{mmol} / \mathrm{L}$, or specific treatment, low HDL $(<1.3 \mathrm{mmol} / \mathrm{L})$ or specific treatment, high blood pressure (SBP $>130$ and/or DBP $\geq 85 \mathrm{mmHg}$ ) or treatment of diagnosed hypertension, and FPG $\geq 5.6 \mathrm{mmol} / \mathrm{L}$ or T2DM. According to the JIS criteria, the diagnosis of MetS was established as the presence of any three of the following criteria: 1) central adiposity (WC $\geq 80 \mathrm{~cm}$ ), 2) TG $\geq 1.7 \mathrm{mmol} / \mathrm{L}$, or specific treatment, 3 ) $\mathrm{HDL}<1.3 \mathrm{mmol} / \mathrm{L}$ or specific treatment, 4) SBP $\geq 130$ and/or DBP $\geq 85 \mathrm{mmHg}$ or treatment of diagnosed hypertension, and 5) FPG $\geq 5.6 \mathrm{mmol} / \mathrm{L}$ or previously diagnosed $\mathrm{T} 2 \mathrm{DM}$ that is under treatment.

The study was approved by the Institutional Ethical Committees and written consent was obtained from all subjects.

\section{Statistical analyses}

Statistical analysis was performed using the Statistical Package for the Social Sciences software (SPSS, version 17.0; SPSS Inc, Chicago, IL, USA). As there were significant differences in age and BMI between PCOS phenotypes and control group, all statistical analyses were adjusted for age and BMI, except BMI and WC, which were adjusted only for age. Accordingly, results are presented as ANCOVAmean (95\% confidence interval (95\% CI)). Normality of data distribution of continuous variables was tested by the Kolmogorov-Smirnov test. Data with skewness or kurtosis were logarithmically transformed in order to achieve normal distribution.

Binary logistic regression analyses were performed in order to analyze the determinants of MetS defined by NCEP-ATP III criteria and LAP in each PCOS phenotype. Only significant determinants from univariate analyses entered multivariate analyses, performed by Forward LR method.

Receiver operating characteristic (ROC) curves were generated for each continuous variable to identify the performance of classic determinants of MetS, as well as LAP. The areas under the curves (AUCs) are provided with standard error of mean (s.E.M.) and 95\% confidence intervals $(95 \% \mathrm{CI})$. ROC curves, a plot of the sensitivity (SEN) (true positive) versus 1-specificity (SP) (false positive) for each potential indicator tested, determine the ability of a screening measure to correctly identify individuals based on their classification by a reference test. 


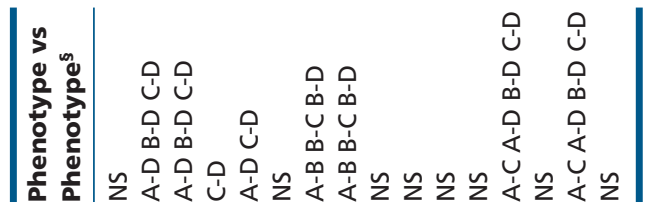

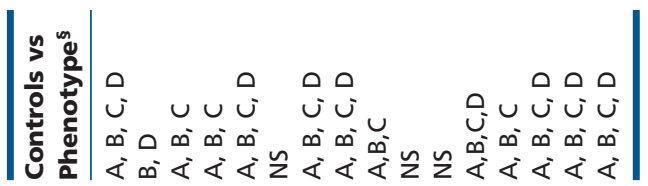

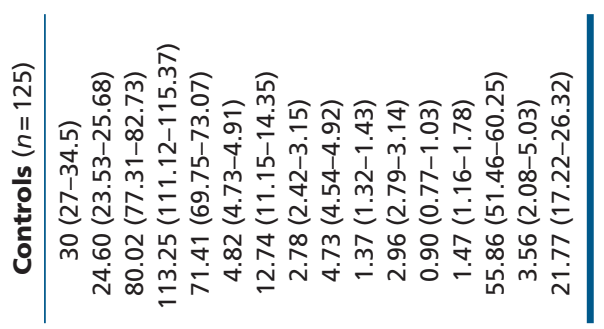

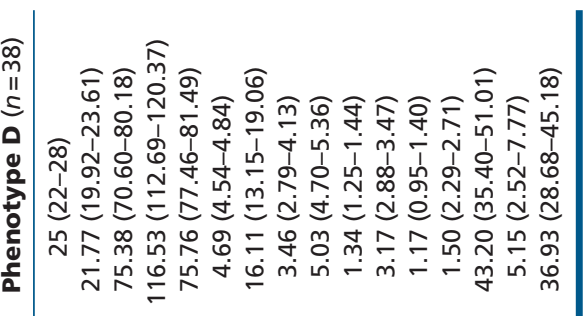

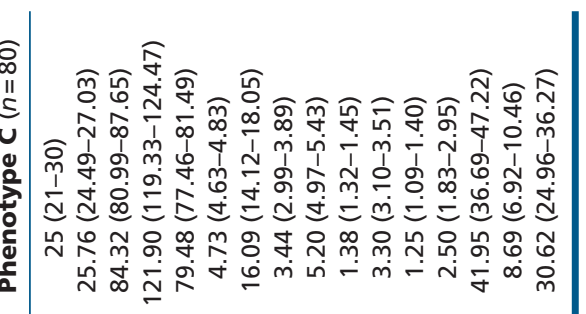

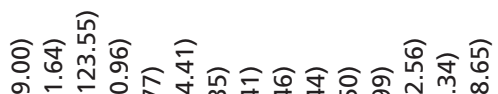
হं்

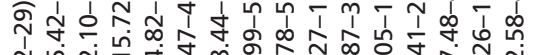

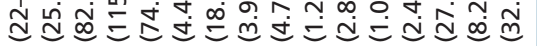

ป స్ N
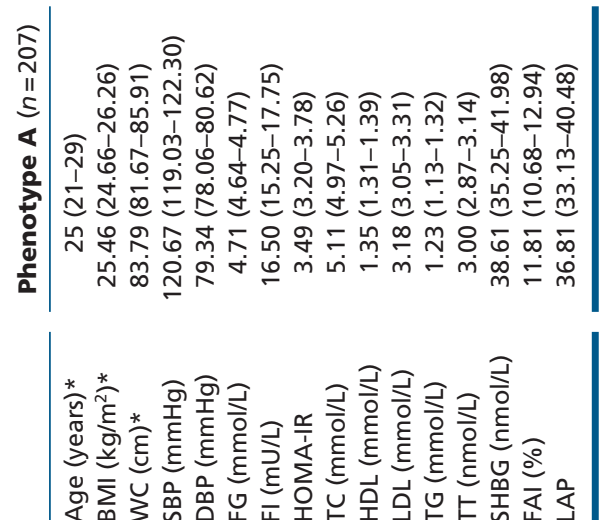

Values for each AUC can be between 0 and 1, with a value of 0.5 indicating that the diagnostic test is no better than chance. We considered that the parameters possessed an accurate diagnostic sensitivity when the AUC value was >0.75 (32). We defined the best cut-off value as the value with the highest proportion of positives and negatives classified correctly by the test. A $P$ value less than 0.05 was considered statistically significant.

\section{Results}

In our PCOS group, 207 (57\%) had phenotype A, 40 (11\%) phenotype B, 80 (22\%) phenotype C and 38 (10\%) phenotype D. Clinical parameters are presented in Table 1.

PCOS phenotypes in comparison to controls showed BMI to be significantly higher for phenotype $B$ and significantly lower for phenotype D, while it was similar for phenotypes $\mathrm{A}$ and $\mathrm{C}$. At the same time phenotypes $\mathrm{A}$ and $\mathrm{C}$, as well as phenotype $\mathrm{B}$, had greater WC. Although women with phenotype D had lower BMI, their WC was similar as in controls. All PCOS phenotypes had higher DBP, TG, fasting insulin, HOMA-IR, FAI and LAP, and lower SHBG in comparison to controls, while there were no differences in fasting glucose, HDL and LDL. Hyperandrogenic phenotypes $(\mathrm{A}, \mathrm{B}, \mathrm{C})$ in comparison to controls had higher SBP, TC and TT.

Comparison between PCOS phenotypes did not show differences in fasting glucose, lipids, SHBG and LAP. While hyperandrogenic phenotypes had the same BMI and WC, phenotype B showed the highest fasting insulin and HOMA-IR. At the same time, phenotype $\mathrm{D}$ had lower BMI, WC, TT and FAI in comparison to hyperandrogenic phenotypes.

Impaired fasting glucose (IFG) was more prevalent in PCOS than controls $(1.4 \%$ vs $0 \%, P=0.045)$. Of four PCOS women with IFG, two (50\%) had phenotype A, one (25\%) had phenotype C and one (25\%) had phenotype D. Impaired glucose tolerance (IGT) was confirmed in ten $(2.7 \%)$ women with PCOS. IGT was equally prevalent among hyperandrogenic PCOS phenotypes (seven (1.9\%) with phenotype A, two (0.5\%) with phenotype $\mathrm{B}$ and one $(0.3 \%)$ with phenotype $\mathrm{C}, P=0.440)$ as well between all PCOS phenotypes and controls $(P=0.523)$. Healthy controls did not show dysregulation of glucose metabolism, and none of the PCOS women had T2DM.

Age and BMI adjusted prevalence of MetS according to different international definitions are presented in Table 2. After age adjustment, all hyperandrogenic phenotypes in comparison to controls had higher 
prevalence of MetS defined by NCEP-ATP III and JIS criteria, while A and B phenotypes had higher prevalence when IDF criteria were used. After combined age and BMI adjustment, only phenotypes A and B had higher prevalence of MetS when IDF and JIS criteria were used, while all PCOS phenotypes had higher prevalence of MetS when NCEP-ATP III criteria were used.

In comparison to hyperandrogenic phenotypes, phenotype D had the lowest age adjusted prevalence of MetS when IDF and JIS criteria were used, but it differed only from phenotypes A and B when NCEP-ATP III criteria were used. After combined age and BMI adjustment was applied, all phenotypes had the same prevalence of MetS, irrespective of criteria used.

When the prevalence of the standard individual components for the definition of MetS (WC $\geq 80 \mathrm{~cm}$, $\mathrm{HDL}<1.29 \mathrm{mmol} / \mathrm{L}, \mathrm{FG} \geq 5.6 \mathrm{mmol} / \mathrm{L}$ and $\mathrm{FG} \geq 6.1 \mathrm{mmol} / \mathrm{L}$ ) was analyzed with combined age and BMI adjustment, there were no differences between PCOS phenotypes and controls, as well among PCOS phenotypes (data not shown). As PCOS women and controls had similar body weight and BMI, and no different prevalence of $\mathrm{WC} \geq 80 \mathrm{~cm}$ as a criterion for IDF and JIS definitions, we decided to use only NCEP-ATP III criteria for further analyses.

Univariate logistic regression analyses showed that LAP was a significant determinant of MetS in all PCOS phenotypes (Table 3). Additionally, standard MetS criteria were significant determinants only in phenotype A (Table 3). In order to avoid problem with collinearity, we omitted WC and TG from multivariate logistic regression analyses, and showed that LAP was an independent determinant of MetS defined by NCEP-ATP III criteria in all phenotypes. Additional independent determinants of MetS were DBP in phenotype A, and SBP in phenotype $\mathrm{C}$ (Table 3 ).

ROC curve analysis showed that LAP had the highest diagnostic accuracy for determination of MetS by NCEPATP III criteria in all PCOS phenotypes (Table 4). For phenotype A, LAP cut-off value of 30.63 had SN: 100\%, SP: 81\%, PPV: 59\%, NPV: 100\%. For phenotype B, LAP cut-off value of 39.65 had SN: 100\%, SP: 83\%, PPV: 73.3\%, NPV: $100 \%$. For phenotype C, LAP cut-off value of 24.40 had SN: 100\%, SP: 75\%, PPV: 50\%, NPV: 100\%. For phenotype D, LAP cut-off value of 25.49 had SN: $100 \%$, SP: $87.5 \%$, PPV: 33\%, NPV: $100 \%$.

\section{Discussion}

The results of our study have confirmed that compared with standard determinants of MetS, lipid accumulation

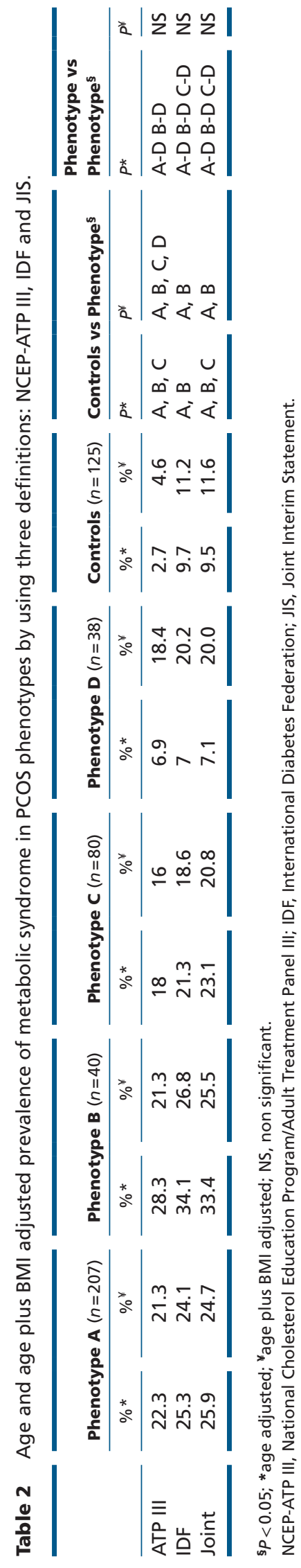

www.eje-online.org 
product has the highest diagnostic accuracy in predicting MetS in Caucasian PCOS population and in each phenotype. Moreover, we have confirmed that both hyperandrogenic and non-hyperandrogenic PCOS phenotypes carry a similar metabolic risk.

MetS represents a collection of cardiometabolic risk factors that expose the subject to a five times higher risk of developing type 2 diabetes mellitus (T2DM) (33), and a two times higher risk of cardiovascular morbidity and mortality (34). Moreover, the risk of atherosclerosis and cardiovascular diseases grows with an increasing number of MetS determinants (35). The prevalence of MetS in PCOS women does show ethnic variation, but it is $2-4$ times higher than that in healthy women (36). As the prevalence of MetS rises with BMI and age (37), it could be presumed that women with PCOS have increased cardiometabolic risks during their reproductive period and menopause, although longitudinal studies are lacking.

There is emerging evidence that PCOS phenotypes differ in anthropometric, hormonal and metabolic characteristics (2-5), which points to diverse cardiometabolic risks and consequences the phenotypes may experience. A few studies that evaluated metabolic characteristics of PCOS phenotypes suggested that classic phenotypes $\mathrm{A}$ and $\mathrm{B}$ have a worse metabolic profile than phenotypes $C$ and $D(6-8,20)$, while some studies showed either no differences between phenotypes (38) or a higher MetS prevalence in phenotype $\mathrm{D}$ in comparison to the hyperandrogenic phenotypes (39). Obesity is among the most important modifiers of phenotypic expression of PCOS. However, even normal-weighted women with PCOS have increased abdominal/visceral adipose tissue, which correlates with insulin resistance, dyslipidemia, hypertension and MetS (40). In accordance with the fact that WC is a marker of abdominal/visceral adiposity (41), we have shown that all PCOS phenotypes have increased abdominal adiposity in comparison to healthy women, regardless of age.

Dyslipidemia is a common metabolic consequence of both obesity and PCOS $(18,42)$. In our population, only hyperandrogenic phenotypes had increased TC, while all phenotypes had increased TG in comparison to controls, regardless of age and BMI. As insulin is the main regulator of SHBG and TG production (43), their respective low and high levels in all analyzed PCOS phenotypes are in accordance with the increased IR in all phenotypes, regardless of age and BMI. Our results are in line with other studies showing the same lipid levels in all phenotypes after age and BMI adjustment $(4,6,38,44,45)$. Interestingly, all our PCOS phenotypes and controls

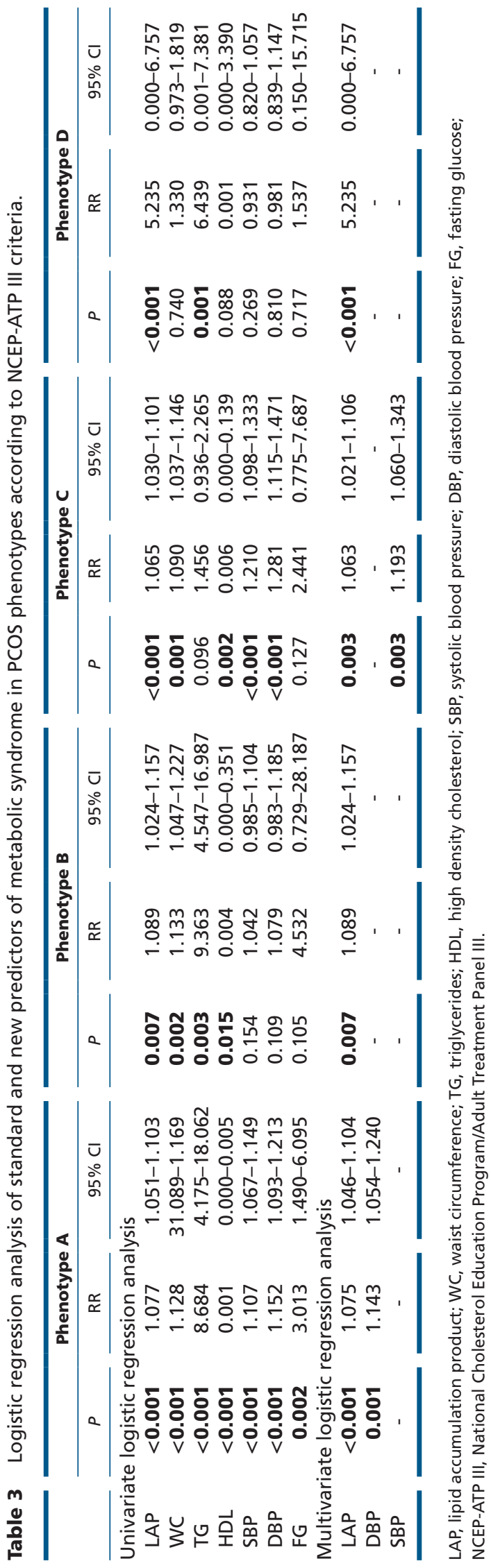


had high prevalence of low HDL after age adjustment (range 33-58\%), as well as after combined age and BMI adjustment (range 36-51\%), exposing our population of PCOS women to a more pronounced cardiovascular risk.

Our PCOS women with phenotype B were significantly more insulin-resistant and hyperinsulinemic in comparison to other phenotypes, regardless of age and BMI. Some studies showed higher IR in hyperandrogenic PCOS phenotypes $(8,20)$.

The results regarding the prevalence of dysglycemia (impaired fasting glucose, impaired glucose tolerance and T2DM) in analyzed PCOS women are comparable to the results of other authors $(6,46)$. Our study was designed as a cross-sectional on four clinical PCOS phenotypes that combine hyperandrogenic and non-hyperandrogenic women. Hence, we do not exclude the possibility that our cohort of PCOS women are at risk of developing T2DM in their future life. Moreover, the deterioration of glucose tolerance toward T2DM could be expected among our cohort of subjects with substantial proportion of MetS, which is considered as an independent predictor of T2DM (47). In effect, it has been shown that 30\% to $40 \%$ of subjects with normal fasting glucose and normal glucose tolerance could have beta-cell dysfunction and insulin resistance for a long period of time before dysglycemia would manifest $(48,49)$.

As far as we know, this is the first study that has analyzed age and combined age and BMI adjusted prevalence of MetS in four classical PCOS phenotypes using three different international criteria. In hyperandrogenic phenotypes, lower MetS prevalence defined by NCEP-ATP III criteria compared with other two definitions is the result of a different cut-off level used for WC criterion.

The results regarding metabolic outcomes in PCOS phenotypes A and B are similar among studies $(44,50)$. However, our women with phenotype C demonstrate the same metabolic risks prevalence (hyperlipidemia, hypertension, insulin resistance) after age and BMI adjustment as women with phenotypes $\mathrm{A}$ and $\mathrm{B}$. Moreover, PCOS women with phenotype D had the same age adjusted MetS prevalence irrespective of international criteria used, as well as a higher age and BMI adjusted MetS prevalence when NCEP-ATP III criteria were applied. Our results suggest that BMI adjustment abolishes the difference between hyperandrogenic and non-hyperandrogenic phenotypes, and that even the non-hyperandrogenic phenotype $\mathrm{D}$ is characterized by an unfavorable metabolic profile.

It is proposed that LAP, a simple clinical marker of lipid over-accumulation in adults, could be used as a 
marker of IR, MetS, cardiovascular risk and mortality in non-PCOS populations $(13,28,51)$ as well as in PCOS population $(15,16,52)$. Previously we showed that LAP was the strongest predictor of MetS defined by three different international criteria in Caucasian women with PCOS (19). In the present study, we have confirmed that LAP is an independent predictor of MetS in each PCOS phenotype. ROC curve analysis showed that of all standard determinants of MetS, LAP had the best discriminative ability for MetS in all PCOS phenotypes as well in controls. Although LAP includes TG and WC, those MetS determinants had lower discriminative power than LAP. All PCOS phenotypes showed higher LAP in comparison to healthy controls after age and BMI adjustment, but without any difference among the phenotypes. These findings are in accordance with MetS prevalence in our PCOS cohort defined by NCEPATP III criteria.

Different LAP cut-off values obtained for each PCOS phenotype with similar SN and SP are in accordance with different metabolic characteristics of the phenotypes. The main value of LAP as a predictor of MetS yields the NPV of $100 \%$ for all proposed cut-offs. Accordingly, our results imply that LAP is a simple time-saving clinical marker for the accurate evaluation of MetS defined by NCEP-ATP III criteria. Therefore, LAP could be used in the routine clinical practice in metabolically vulnerable patients such as women with PCOS, as well as for the evaluation of MetS in PCOS phenotypes.

We are aware of the fact that the use of radioimmunoassay for the analysis of total testosterone concentration is a limitation of our study. While the characteristics of liquid chromatography-tandem mass spectrometry (LC-MS/MS) make steroid hormone analysis a more favorable method for the differentiation of women with $\operatorname{PCOS}(53,54)$, it is expensive for extensive clinical application and is not readily available in the clinical setting in our country. Moreover, a recent study has concluded that the total testosterone measurement using radioimmunoassay and LC-MS/MS differentiated PCOS women from controls in a similar fashion (55).

In conclusion, we have found that LAP is an independent, simple and accurate clinical determinant of the existence of MetS in all phenotypes of our analyzed PCOS population. Moreover, we have confirmed that all PCOS phenotypes, including the non-hyperandrogenic ones, are metabolically susceptible, and carry the potential for the development of abdominal obesity, insulin resistance and cardiovascular morbidity.
Declaration of interest

The authors declare that there is no conflict of interest that could be perceived as prejudicing the impartiality of the research reported.

\section{Funding}

This work was supported by grants 41009 and 175032 from the Serbian Ministry of Science and Education.

\section{Author contribution statement}

D M and I B-A contributed to the conception and design of the study, to the acquisition, analysis and interpretation of data and to drafting the article. D I, J B-M, T B, D V-M, B K-K, O S and Z A contributed to the acquisition of data and revised the article critically for important intellectual content. N M contributed to the statistical analysis and interpretation of data. All authors gave their final approval of the version to be published.

\section{References}

1 Conway GS, Dewailly D, Diamanti-Kandarakis E, Escobar Morreale H, Franks S, Gambineri A, Kelestimur F, Macut D, Micic D, Pasquali R et al. The polycystic ovary syndrome: an endocrinological perspective from the European Society of Endocrinology. European Journal of Endocrinology 2014171 489-498. (doi:10.1530/EJE-14-0252)

2 Panidis D, Tziomalos K, Misichronis G, Papadakis E, Betsas G, Katsikis I \& Macut D. Insulin resistance and endocrine characteristics of the different phenotypes of polycystic ovary syndrome: a prospective study. Human Reproduction 201227 541-549. (doi:10.1093/humrep/der418)

3 Pehlivanov B \& Orbetzova M. Characteristics of different phenotypes of polycystic ovary syndrome in a Bulgarian population. Gynecological Endocrinology 200723 604-609. (doi:10.1080/09513590701536246)

4 Chae SJ, Kim JJ, Choi YM, Hwang KR, Jee BC, Ku SY, Suh CS, Kim SH, Kim JG \& Moon SY. Clinical and biochemical characteristics of polycystic ovary syndrome in Korean women. Human Reproduction 200823 1924-1931. (doi:10.1093/humrep/den239)

5 Zhang HY, Zhu FF, Xiong J, Shi XB \& Fu SX. Characteristics of different phenotypes of polycystic ovary syndrome based on the Rotterdam criteria in a large-scale Chinese population. BJOG 2009 116 1633-1639. (doi:10.1111/j.1471-0528.2009.02347.x)

6 Welt CK, Gudmundsson JA, Arason G, Adams J, Palsdottir H, Gudlaugsdottir G, Ingadottir G \& Crowley WF. Characterizing discrete subsets of polycystic ovary syndrome as defined by the Rotterdam criteria: the impact of weight on phenotype and metabolic features. Journal of Clinical Endocrinology \& Metabolism $2006914842-4848$. (doi:10.1210/jc.2006-1327)

7 Diamanti-Kandarakis E \& Dunaif A. Insulin resistance and the polycystic ovary syndrome revisited: an update on mechanisms and implications. Endocrine Review 201233 981-1030. (doi:10.1210/ er.2011-1034)

8 Daskalopoulos G, Karkanaki A, Piouka A, Prapas N, Panidis D, Gkeleris P \& Athyros VG. Excess metabolic and cardiovascular risk is not manifested in all phenotypes of polycystic ovary syndrome: implications for diagnosis and treatment. Current Vascular Pharmacology 201513 788-800. (doi:10.2174/1567201812666150120 163025)

9 Randeva HS, Tan BK, Weickert MO, Lois K, Nestler JE, Sattar N \& Lehnert H. Cardiometabolic aspects of the polycystic ovary syndrome. Endocrine Review 201233 812-841. (doi:10.1210/er.2012-1003)

10 Azziz R, Marin C, Hoq L, Badamgarav E \& Song P. Health care-related economic burden of the polycystic ovary syndrome during the 
reproductive life span. Journal of Clinical Endocrinology \& Metabolism 200590 4650-4658. (doi:10.1210/jc.2005-0628)

11 Mott MM, Kitos NR \& Coviello AD. Practice patterns in screening for metabolic disease in women with PCOS of diverse race-ethnic backgrounds. Endocrine Practice 201420 855-863. (doi:10.4158/ EP13414.OR)

12 Legro RS, Arslanian SA, Ehrmann DA, Hoeger KM, Murad MH, Pasquali R \& Welt CK. Diagnosis and treatment of polycystic ovary syndrome: an Endocrine Society clinical practice guideline. Journal of Clinical Endocrinology \& Metabolism 201398 4565-4592. (doi:10.1210/ jc.2013-2350)

13 Taverna MJ, Martinez-Larrad MT, Frechtel GD \& Serrano-Rios M. Lipid accumulation product: a powerful marker of metabolic syndrome in healthy population. European Journal of Endocrinology 2011164 559-567. (doi:10.1530/EJE-10-1039)

14 Bozorgmanesh M, Hadaegh F \& Azizi F. Diabetes prediction, lipid accumulation product, and adiposity measures; 6-year follow-up: Tehran lipid and glucose study. Lipids in Health and Disease 2010945. (doi:10.1186/1476-511X-9-45)

15 Wiltgen D, Benedetto IG, Mastella LS \& Spritzer PM. Lipid accumulation product index: a reliable marker of cardiovascular risk in polycystic ovary syndrome. Human Reproduction 200924 1726-1731. (doi:10.1093/humrep/dep072)

16 Wehr E, Gruber HJ, Giuliani A, Moller R, Pieber TR \& ObermayerPietsch B. The lipid accumulation product is associated with impaired glucose tolerance in PCOS women. Journal of Clinical Endocrinology \& Metabolism 201196 E986-E990. (doi:10.1210/jc.2011-0031)

17 Xiang S, Hua F, Chen L, Tang Y, Jiang X \& Liu Z. Lipid accumulation product is related to metabolic syndrome in women with polycystic ovary syndrome. Experimental and Clinical Endocrinology \& Diabetes 2013121 115-118. (doi:10.1055/s-0032-1333261)

18 Macut D, Tziomalos K, Bozic-Antic I, Bjekic-Macut J, Katsikis I, Papadakis E, Andric Z \& Panidis D. Non-alcoholic fatty liver disease is associated with insulin resistance and lipid accumulation product in women with polycystic ovary syndrome. Human Reproduction 201631 1347-1353. (doi:10.1093/humrep/dew076)

19 Macut D, Bozic Antic I, Bjekic-Macut J, Panidis D, Tziomalos K, Vojnovic Milutinovic D, Stanojlovic O, Kastratovic-Kotlica B, Petakov M \& Milic N. Lipid accumulation product is associated with metabolic syndrome in women with polycystic ovary syndrome. Hormones 201615 35-44. (doi:10.14310/horm.2002.1592)

20 Daan NM, Louwers YV, Koster MP, Eijkemans MJ, de Rijke YB, Lentjes EW, Fauser BC \& Laven JS. Cardiovascular and metabolic profiles amongst different polycystic ovary syndrome phenotypes: who is really at risk? Fertility and Sterility $2014 \mathbf{1 0 2} 1444-1451$ e1443. (doi:10.1016/j.fertnstert.2014.08.001)

21 Rotterdam ESHRE/ASRM-Sponsored PCOS consensus workshop group. Revised 2003 consensus on diagnostic criteria and longterm health risks related to polycystic ovary syndrome (PCOS). Human Reproduction 200419 41-47. (doi:10.1093/humrep/deh098)

22 Ferriman D \& Gallwey JD. Clinical assessment of body hair growth in women. Journal of Clinical Endocrinology \& Metabolism 196121 1440-1447. (doi:10.1210/jcem-21-11-1440)

23 Escobar-Morreale HF, Carmina E, Dewailly D, Gambineri A, Kelestimur F, Moghetti P, Pugeat M, Qiao J, Wijeyaratne CN, Witchel SF et al. Epidemiology, diagnosis and management of hirsutism: a consensus statement by the Androgen Excess and Polycystic Ovary Syndrome Society. Human Reproduction Update 2012 18 146-170. (doi:10.1093/humupd/dmr042)

24 Mathur RS, Moody LO, Landgrebe S \& Williamson HO. Plasma androgens and sex hormone-binding globulin in the evaluation of hirsute females. Fertility and Sterility 198135 29-35. (doi:10.1016/ S0015-0282(16)45254-4)

25 Macut D, Damjanovic S, Panidis D, Spanos N, Glisic B, Petakov M, Rousso D, Kourtis A, Bjekic J \& Milic N. Oxidised low-density lipoprotein concentration - early marker of an altered lipid metabolism in young women with PCOS. European Journal of Endocrinology 2006155 131-136. (doi:10.1530/eje.1.02187)

26 Friedewald WT, Levy RI \& Fredrickson DS. Estimation of the concentration of low-density lipoprotein cholesterol in plasma, without use of the preparative ultracentrifuge. Clinical Chemistry 1972 18 499-502.

27 Matthews DR, Hosker JP, Rudenski AS, Naylor BA, Treacher DF \& Turner RC. Homeostasis model assessment: insulin resistance and beta-cell function from fasting plasma glucose and insulin concentrations in man. Diabetologia 198528 412-419. (doi:10.1007/ BF00280883)

28 Kahn HS. The "lipid accumulation product" performs better than the body mass index for recognizing cardiovascular risk: a populationbased comparison. BMC Cardiovascular Disorders 2005526. (doi:10.1186/1471-2261-5-26)

29 Third Report of the National Cholesterol Education Program (NCEP) Expert Panel on Detection, Evaluation, and Treatment of High Blood Cholesterol in Adults (Adult Treatment Panel III) final report. Circulation 2002106 3143-3421.

30 Alberti KG, Zimmet P \& Shaw J. The metabolic syndrome--a new worldwide definition. Lancet 2005366 1059-1062. (doi:10.1016/ S0140-6736(05)67402-8)

31 Alberti KG, Eckel RH, Grundy SM, Zimmet PZ, Cleeman JI, Donato KA, Fruchart JC, James WP, Loria CM \& Smith SC Jr. Harmonizing the metabolic syndrome: a joint interim statement of the International Diabetes Federation Task Force on Epidemiology and Prevention; National Heart, Lung, and Blood Institute; American Heart Association; World Heart Federation; International Atherosclerosis Society; and International Association for the Study of Obesity. Circulation 2009120 1640-1645. (doi:10.1161/ CIRCULATIONAHA.109.192644)

32 Hanley JA \& McNeil BJ. A method of comparing the areas under receiver operating characteristic curves derived from the same cases. Radiology 1983148 839-843. (doi:10.1148/radiology.148.3.6878708)

33 Ford ES, Li C \& Sattar N. Metabolic syndrome and incident diabetes: current state of the evidence. Diabetes Care 200831 1898-1904. (doi:10.2337/dc08-0423)

34 Mottillo S, Filion KB, Genest J, Joseph L, Pilote L, Poirier P, Rinfret S, Schiffrin EL \& Eisenberg MJ. The metabolic syndrome and cardiovascular risk a systematic review and meta-analysis. Journal of the American College of Cardiology 201056 1113-1132. (doi:10.1016/j. jacc.2010.05.034)

35 Athyros VG, Mikhailidis DP, Papageorgiou AA, Didangelos TP, Ganotakis ES, Symeonidis AN, Daskalopoulou SS, Kakafika AI \& Elisaf M. Prevalence of atherosclerotic vascular disease among subjects with the metabolic syndrome with or without diabetes mellitus: the METS-GREECE Multicentre Study. Current Medical Research and Opinion 200420 1691-1701. (doi:10.1185/030079904X5599)

36 Moran LJ, Misso ML, Wild RA \& Norman RJ. Impaired glucose tolerance, type 2 diabetes and metabolic syndrome in polycystic ovary syndrome: a systematic review and meta-analysis. Human Reproduction Update 201016 347-363. (doi:10.1093/humupd/ dmq001)

37 Ervin RB. Prevalence of metabolic syndrome among adults 20 years of age and over, by sex, age, race and ethnicity, and body mass index: United States, 2003-2006. National Health Statistics Reports 2009 1-7.

38 Ates S, Sevket O, Sudolmus S, Dane B, Ozkal F, Uysal O \& Dansuk R. Different phenotypes of polycystic ovary syndrome in Turkish women: clinical and endocrine characteristics. Gynecological Endocrinology 201329 931-935. (doi:10.3109/09513590.2013.819082)

39 Zhang J, Fan P, Liu H, Bai H, Wang Y \& Zhang F. Apolipoprotein A-I and B levels, dyslipidemia and metabolic syndrome in south-west Chinese women with PCOS. Human Reproduction 201227 2484-2493. (doi:10.1093/humrep/des191) 
40 Carmina E, Bucchieri S, Esposito A, Del Puente A, Mansueto P, Orio F, Di Fede G \& Rini G. Abdominal fat quantity and distribution in women with polycystic ovary syndrome and extent of its relation to insulin resistance. Journal of Clinical Endocrinology \& Metabolism 2007 92 2500-2505. (doi:10.1210/jc.2006-2725)

41 Ehrmann DA, Liljenquist DR, Kasza K, Azziz R, Legro RS \& Ghazzi MN. Prevalence and predictors of the metabolic syndrome in women with polycystic ovary syndrome. Journal of Clinical Endocrinology \& Metabolism 200691 48-53. (doi:10.1210/jc.2005-1329)

42 Macut D, Bjekic-Macut J \& Savic-Radojevic A. Dyslipidemia and oxidative stress in PCOS. Frontiers of Hormone Research 201340 51-63. (doi:10.1159/000341683)

43 Nestler JE, Powers LP, Matt DW, Steingold KA, Plymate SR, Rittmaster RS, Clore JN \& Blackard WG. A direct effect of hyperinsulinemia on serum sex hormone-binding globulin levels in obese women with the polycystic ovary syndrome. Journal of Clinical Endocrinology \& Metabolism 199172 83-89. (doi:10.1210/jcem-72-1-83)

44 Barber TM, Wass JA, McCarthy MI \& Franks S. Metabolic characteristics of women with polycystic ovaries and oligoamenorrhoea but normal androgen levels: implications for the management of polycystic ovary syndrome. Clinical Endocrinology 200766 513-517. (doi:10.1111/j.1365-2265.2007.02764.x)

45 Kauffman RP, Baker TE, Baker VM, DiMarino P \& Castracane VD. Endocrine and metabolic differences among phenotypic expressions of polycystic ovary syndrome according to the 2003 Rotterdam consensus criteria. American Journal of Obstetrics \& Gynecology 2008 198670 e671-e677; discussion 670 e677-e610. (doi:10.1016/j. ajog.2008.01.037)

46 Luque-Ramirez M, Alpanes M \& Escobar-Morreale HF. The determinants of insulin sensitivity, beta-cell function, and glucose tolerance are different in patients with polycystic ovary syndrome than in women who do not have hyperandrogenism. Fertility and Sterility 201094 2214-2221. (doi:10.1016/j.fertnstert.2009.11.049)

47 Lorenzo C, Okoloise M, Williams K, Stern MP \& Haffner SM. The metabolic syndrome as predictor of type 2 diabetes: the San Antonio heart study. Diabetes Care 200326 3153-3159. (doi:10.2337/ diacare.26.11.3153)
48 Gastaldelli A, Ferrannini E, Miyazaki Y, Matsuda M \& DeFronzo RA. Beta-cell dysfunction and glucose intolerance: results from the San Antonio metabolism (SAM) study. Diabetologia 200447 31-39. (doi:10.1007/s00125-003-1263-9)

49 Ferrannini E, Gastaldelli A, Miyazaki Y, Matsuda M, Mari A \& DeFronzo RA. beta-Cell function in subjects spanning the range from normal glucose tolerance to overt diabetes: a new analysis. Journal of Clinical Endocrinology \& Metabolism 200590 493-500. (doi:10.1210/ jc.2004-1133)

50 Shroff R, Syrop CH, Davis W, Van Voorhis BJ \& Dokras A. Risk of metabolic complications in the new PCOS phenotypes based on the Rotterdam criteria. Fertility and Sterility 200788 1389-1395. (doi:10.1016/j.fertnstert.2007.01.032)

51 Ioachimescu AG, Brennan DM, Hoar BM \& Hoogwerf BJ. The lipid accumulation product and all-cause mortality in patients at high cardiovascular risk: a PreCIS database study. Obesity 201018 1836-1844. (doi:10.1038/oby.2009.453)

52 Hosseinpanah F, Barzin M, Erfani H, Serahati S, Ramezani Tehrani F $\&$ Azizi F. Lipid accumulation product and insulin resistance in Iranian PCOS prevalence study. Clinical Endocrinology 201481 52-57. (doi:10.1111/cen.12287)

53 Barth JH, Field HP, Yasmin E \& Balen AH. Defining hyperandrogenism in polycystic ovary syndrome: measurement of testosterone and androstenedione by liquid chromatography-tandem mass spectrometry and analysis by receiver operator characteristic plots. European Journal of Endocrinology 2010162 611-615. (doi:10.1530/EJE-09-0741)

54 Janse F, Eijkemans MJ, Goverde AJ, Lentjes EG, Hoek A, Lambalk CB, Hickey TE, Fauser BC \& Norman RJ. Assessment of androgen concentration in women: liquid chromatography-tandem mass spectrometry and extraction RIA show comparable results. European Journal of Endocrinology 2011165 925-933. (doi:10.1530/EJE-11-0482)

55 Salameh WA, Redor-Goldman MM, Clarke NJ, Mathur R, Azziz R \& Reitz RE. Specificity and predictive value of circulating testosterone assessed by tandem mass spectrometry for the diagnosis of polycystic ovary syndrome by the National Institutes of Health 1990 criteria. Fertility and Sterility 2014101 1135-1141 e1132. (doi:10.1016/j. fertnstert.2013.12.056)

Received 10 September 2016 Accepted 15 September 2016 